\title{
Broken Mirror Symmetry of Tracer's Trajectories in Turbulence
}

\author{
S. Angriman $\odot,{ }^{1, *}$ P. J. Cobelli $\odot,{ }^{1}$ M. Bourgoin, ${ }^{2}$ S. G. Huisman $\odot,{ }^{3}$ R. Volk $\odot,{ }^{2}$ and P. D. Mininni ${ }^{1}$ \\ ${ }^{1}$ Universidad de Buenos Aires, Facultad de Ciencias Exactas y Naturales, Departamento de Física, \& IFIBA, \\ CONICET, Ciudad Universitaria, Buenos Aires 1428, Argentina \\ ${ }^{2}$ Université Lyon, ENS de Lyon, Université Claude Bernard, CNRS, Laboratoire de Physique, \\ 46 Allée d'Italie F-69342 Lyon, France \\ ${ }^{3}$ Physics of Fluids Group, Max Planck UT Center for Complex Fluid Dynamics, Faculty of Science and \\ Technology, MESA+ Institute and J.M. Burgers Centre for Fluid Dynamics, University of Twente, \\ P.O. Box 217, 7500 AE Enschede, Netherlands
}

(Received 17 March 2021; revised 5 July 2021; accepted 12 November 2021; published 17 December 2021)

\begin{abstract}
Topological properties of physical systems play a crucial role in our understanding of nature, yet their experimental determination remains elusive. We show that the mean helicity, a dynamical invariant in ideal flows, quantitatively affects trajectories of fluid elements: the linking number of Lagrangian trajectories depends on the mean helicity. Thus, a global topological invariant and a topological number of fluid trajectories become related, and we provide an empirical expression linking them. The relation shows the existence of long-term memory in the trajectories: the links can be made of the trajectory up to a given time, with particles positions in the past. This property also allows experimental measurements of mean helicity.
\end{abstract}

DOI: 10.1103/PhysRevLett.127.254502

In recent years, broken symmetries and topology have played an increasing role in physics. Examples are topological phase transitions [1], topological charges in condensed matter [2], applications in quantum field theory [3], electromagnetism [4], DNA [5], and chromosome organization [6]. In fluid dynamics, three-dimensional (3D) barotropic flows have an ideal invariant of topological nature. Helicity, the inner product between the Eulerian velocity $\mathbf{u}$ and the vorticity $\nabla \times \mathbf{u}$, integrated over the fluid volume $V, H=V^{-1} \int \mathbf{u} \cdot(\boldsymbol{\nabla} \times \mathbf{u}) d V$, is proportional to the Gauss linking number of vorticity field lines [7,8], and measures their linkage and knottedness. Helicity is the only integral invariant of volume-preserving transformations [9]. Moreover, its presence indicates the flow has no mirror symmetry (i.e., it is chiral). Helicity is relevant in astrophysical [10-13] and geophysical flows [14-16], in superfluids and Bose-Einstein condensates [17-22], and in swirling [23] and rotating [24] flows. In active fluids, it can generate a helicity-driven inverse energy cascade (i.e., a self-similar transfer of energy to larger scales) [25]. In turbulence, the symmetry breaking introduced by nonzero helicity affects the statistical properties of the energy cascade, and leads to strongly depleted energy transfers between scales $[8,26]$, or to a change in the energy transfer direction $[25,27]$.

Characterizing the topology of a vector field from a discrete set of measurements constitutes a cross-cutting challenge concerning several areas, such as surface reconstruction, deep learning, time series classification, and chaotic attractor embeddings [28]. In fluid dynamics, helicity, although theoretically appealing, is hard to measure. Experimental estimations employ pointwise measurements of velocity and vorticity (which are incomplete as helicity is a global quantity), or use linking numbers in flows simple enough that vorticity field lines can be identified $[29,30]$. Helicoidal particles were also devised to estimate local flow chirality [31]. Despite these attempts, measurements in the fully turbulent regime remain difficult, resulting in discussions on its conservation [32,33].

Here, we show that the broken mirror symmetry associated with helicity affects the connectivity of fluid elements trajectories, generating linkages between their long-time history. This is accomplished by combining simulations of homogeneous and isotropic turbulence (HIT) and of Taylor-Green (TG) flows at different Reynolds numbers, with laboratory experiments of mirror-symmetric HIT and of chiral von Kármán (VK) flows. The robustness of the results allows us to define a new volumetric measurement of helicity using the particle linking number, providing access to global quantification of helicity in experiments.

Definition of the linking number of fluid elements trajectories.-Does the number of links between tracers' trajectories constitute a proxy of the helicity of the underlying flow? Particles' trajectories do not form, in general, closed loops. Even if some closed orbits exist under artificial (e.g., periodic) boundary conditions, laboratory measurements extend for a finite time and consist of short trajectories, spanning from a fraction to a few flow correlation times, so there is no notion of knottedness. Still, we can define an average linking number between any set of $3 \mathrm{D}$ curves as the mean value of the signed apparent 

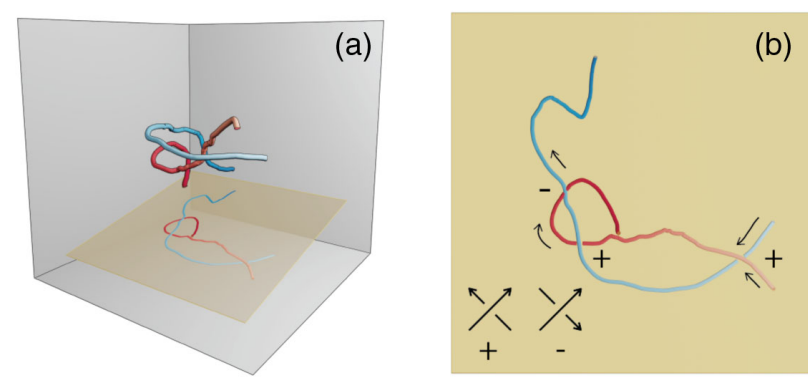

FIG. 1. Definition of apparent crossing. A pair of experimental $3 \mathrm{D}$ trajectories (a) are projected onto a 2D plane [bottom of (a), and (b)]. Arrows and color gradients indicate time progression. A crossing is an intersection between the projections and is given by the particles' history; it does not necessarily occur with the particles being near at the same instant. The sign of each crossing is defined by the right-hand rule [(b), bottom]: +1 when an anticlockwise rotation is needed to move from the tip of the arrow on top to the tip of the arrow below, and -1 in the clockwise case. Here, with two tracks projected in one plane, we see one negative and two positive crossings.

crossings in a $P$ number of two-dimensional (2D) projections. We thus consider a time interval $\Delta T$ over which we have measurements, and $N$ tracer trajectories that sample the flow during this interval. To compute the total number of signed crossings, we project the curves onto $P$ differently oriented planes, as if computing the 2D "shadows" of the trajectories. In each shadow, an apparent crossing between two projected curves is defined as their intersection. A crossing may also occur between two different time instants of the same trajectory; these self-crossings are treated identically (we verified that removing self-crossings yields the same results). Note that crossings are not instantaneous crossings between particles, but between their history. The sign of each crossing (i.e., counting it as +1 or -1 ) is given by the right-hand rule (see Fig. 1): we keep track of what trajectory is on top, and in what direction particles moved when going across the vertex. This orientation defines the handedness of the crossing [3].

The normalized crossings $K_{p}$ for the $p$ th projection are defined as $K_{p}=M_{p}^{-1} \Sigma_{i=1}^{M_{p}} \sigma_{i}$, where $M_{p}$ is the total (unsigned) apparent crossings in the $p$ th projection, and $\sigma_{i}= \pm 1$ is the sign of the $i$ th crossing. Then, we define the mean linking number of the $N$ trajectories over the interval $\Delta T$ as the mean of $K_{p}$ over all $P$ projections: $\mathcal{K}=$ $P^{-1} \sum_{p=1}^{P} K_{p}$.

Description of the data.-To study $\mathcal{K}$ and $H$ we consider direct numerical simulations (DNSs) and tracers from particle tracking velocimetry (PTV) in laboratory experiments (see [34] for more details). We use two sets of DNSs $[35,36]$ with resolutions of $256^{3}, 512^{3}$, and $1024^{3}$ points to span different Reynolds numbers. The first consists of DNSs of HIT with correlated random forcing to give a flow with a tunable helicity [37] (we also consider a "HIT 2" simulation with very short forcing correlation time).
Another set of DNSs uses TG forcing [38,39] which mimics, in a periodic domain, multiple cells resembling VK flows, each non-mirror symmetric and with non-null helicity [with alternating signs between the cells, resulting in null total helicity in a $(2 \pi)^{3}$-periodic domain]. In each simulation $10^{6}$ tracers were evolved along with the fluid. Experimental data on tracers trajectories obtained by PTV originates from two experiments: a VK experiment in Buenos Aires [40] generates a helical flow, and the Lagrangian Exploration Module (LEM) in Lyon [41,42] generates mirror-symmetric isotropic turbulence.

For each dataset, $\mathcal{K}$ was computed using sets of trajectories that span a large-scale volume of the flow in nonoverlapping time intervals $\Delta T$ ranging from a fraction to several $\tau_{L}$, with $\tau_{L}$ the Lagrangian correlation time (estimated from the tracers velocity autocorrelation function, or from structure functions in the LEM $[41,43])$. We consider subsets of $N=250$ particles in the DNSs and all available particles in the experiments, with $P=26$ projections whose normal vectors are approximately uniformly distributed over a unit sphere, and given by $\hat{\mathbf{n}}_{p}=(i \hat{\mathbf{x}}+$ $j \hat{\mathbf{y}}+k \hat{\mathbf{z}}) /\left(i^{2}+j^{2}+k^{2}\right)^{1 / 2}$ with $i, j, k \in\{-1,0,1\}$. This number of projections was empirically established as the minimum required to consistently recover the linking number of randomly oriented torus knots.

The relation between the linking number of trajectories and helicity.-We analyze $\mathcal{K}$ as a function of the normalized, dimensionless helicity $\mathcal{H}$

$$
\mathcal{H}=L U^{-2}\langle H\rangle_{\Delta T},
$$

where $\langle\cdot\rangle_{\Delta T}$ indicates time averaging over $\Delta T, U=\left(\left\langle v_{x}^{2}+\right.\right.$ $\left.\left.v_{y}^{2}+v_{z}^{2}\right\rangle\right)^{1 / 2}$ (with $v_{i}$ the components of the tracers' velocity and $\langle\cdot\rangle$ the average over $\Delta T$ and all trajectories) is a measure of the tracers' velocity over $\Delta T$ (to consider possible effects of velocity variations over $\Delta T$ on the helicity), and $L=u \tau_{L}$ is a flow integral scale based on the characteristic one-component rms value of the tracers' velocity $u$ estimated over a long time interval. These choices allow for the estimation of all quantities solely from Lagrangian measurements.

Figure 2 shows $\mathcal{K}$ as a function of $\mathcal{H}$ for DNSs of HIT and for three datasets of the LEM experiment. Error bars represent 95\% confidence intervals (approximately twice the standard deviation of $\mathcal{K}$ ). $\mathcal{K}$ and $\mathcal{H}$ were computed for $\Delta T=\tau_{L}$. For the entire range of $\mathcal{H}$ explored, the data display a linear dependence between the two quantities, irrespective of Reynolds number and flow geometry. Therefore, we propose that these two global, large-scale quantities are related by

$$
\mathcal{K}=\alpha \mathcal{H},
$$

where $\alpha$ is an unknown dimensionless constant. An additive constant is not expected in this relation, as we 


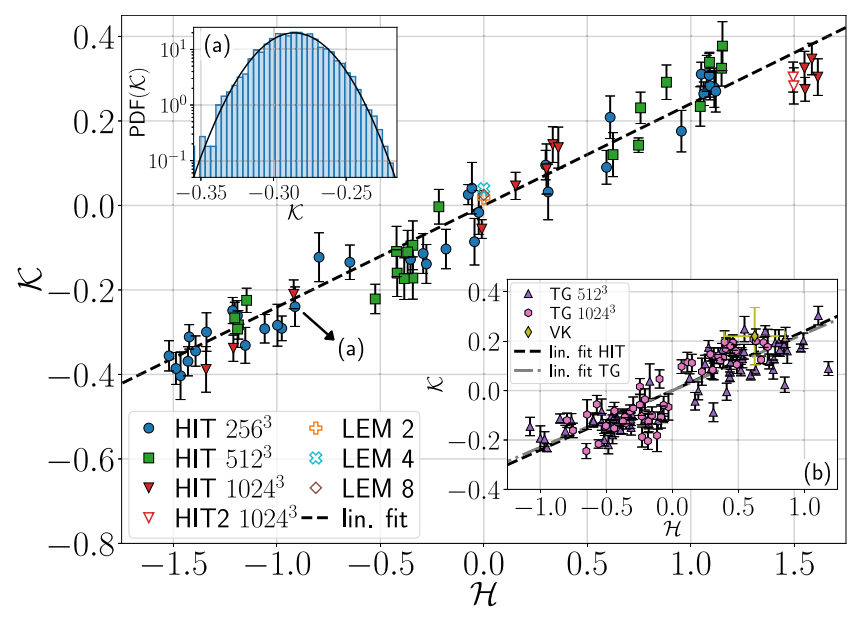

FIG. 2. Linking number of tracers trajectories $\mathcal{K}$ as a function of normalized helicity $\mathcal{H}$ for DNSs of HIT and the LEM experiment. Error bars correspond to $95 \%$ confidence intervals. A leastsquares linear fit taking into account the error bars is shown as a reference. Inset (a) shows the PDF of $\mathcal{K}$ in semilogarithmic scale for different subsets of tracers of the same DNS, indicated by the arrow. A Gaussian distribution with the same mean and standard deviation is shown for comparison. Inset (b) shows $\mathcal{K}$ as a function of $\mathcal{H}$ for the VK experiment, and cells in TG simulations; each of the cells corresponds to a VK-like swirling flow with nonzero mean helicity. A weighted least-squares linear fit for all TG data is shown, and it is compared with the linear fit obtained from HIT.

can assume that a mirror-symmetric flow will have statistically as many +1 crossings as -1 crossings (which is consistent with the data). An error-weighted leastsquares fit using the HIT data yields $\alpha_{\text {HIT }}=0.241 \pm$ 0.006 (95\% confidence level) independently of the Reynolds number (provided a fully developed turbulent state is reached). A dashed straight line with this slope is shown in Fig. 2. We verified that a linear relation as in Eq. (2) holds regardless of the particular choice of $U$ and $L$ employed to normalize $H$ in Eq. (1).

Before discussing the other datasets, note Eq. (2) is robust: it holds for all datasets with small changes in $\alpha$ within errors, and when the number of particles $N$, the time span $\Delta T$, or other parameters are changed, and also when sufficiently large subregions of the flow are considered. To understand how using a finite number of trajectories affects the determination of $\mathcal{K}$ and its error, different subsets of 250 particles were randomly chosen from the $10^{6}$ trajectories traced in each DNS. We see minor variations in the value of $\mathcal{K}$, as shown by the probability distribution function (PDF) of $\mathcal{K}$ for different subsets in a DNS (indicated by the arrow) in Fig. 2(a). The PDF is compatible with a Gaussian distribution and its dispersion is associated with the errors in $\mathcal{K}$ when using a finite number of tracers. Note this PDF does not correspond to local helicity fluctuations: it is a measure of the uncertainty in the determination of $\mathcal{K}$. Using $N=250$ is motivated by the number of trajectories that can be simultaneously observed using PTV in experiments, of the order of the several tens to a few hundreds. Indeed, such value is enough to get a reasonable determination of $\mathcal{K}$, with smaller errors as $N$ is increased. As $\Delta T$ decreases, $N=250$ is still enough to determine $\mathcal{K}$, although with larger error bars. By varying $\Delta T$, reasonable correlation (with the same value of $\alpha$ ) is obtained between $\mathcal{K}$ and $\mathcal{H}$ when $\Delta T \geq \tau_{L} / 5$. For $\Delta T<\tau_{L} / 10$ uncertainties in $\mathcal{K}$ prevent distinction between chiral and nonchiral flows. By increasing $N$ to 1000 , we verified that computing $\mathcal{K}$ over $\Delta T=\tau_{L} / 10$ allows for a distinction between different flow chiralities, but to achieve a correlation between $\mathcal{K}$ and $\mathcal{H}$ (consistent within uncertainties) it is still necessary to use $\Delta T \approx \tau_{L} / 5$. This can be interpreted as a limit on how short a history of the flow is needed to reconstruct its topology. Furthermore, the condition $\Delta T \geq \tau_{L} / 5$ implies that if $\mathcal{K}$ is used to estimate the helicity in a flow as a function of time, $\approx 0.2 \tau_{L}$ is the maximum time cadence for which $\mathcal{K}(t)$ [and thus $\mathcal{H}(t)$ ] can be estimated. Finally, increasing $\Delta T$ (for fixed $N$ ) results in a better agreement between $\mathcal{K} / \alpha$ and $\mathcal{H}$. See [34] for more details on the uncertainty in the determination of $\mathcal{K}$, and on the robustness of the results on $N, \Delta T$ and on the memory of the trajectories.

As previously mentioned, Fig. 2 also shows data from tracers in LEM, an experiment that generates mirrorsymmetric (i.e., zero helicity) HIT. The turbulence generation mechanism is quite different from the DNSs, using multiple impellers instead of random volumetric forcing. Three experimental runs with different Reynolds numbers (labeled LEM 2, LEM 4, and LEM 8) were analyzed. $\mathcal{K}$ was estimated from the signed crossings over $\Delta T=\tau_{L}$ for each run. The mean value of $\mathcal{K}$ for each one is shown in Fig. 2; their values are $\mathcal{K}_{\text {LEM } 2}=0.018 \pm 0.18, \mathcal{K}_{\text {LEM } 4}=$ $0.040 \pm 0.29$, and $\mathcal{K}_{\text {LEM } 8}=0.025 \pm 0.28$ (95\% confidence intervals). Such large fluctuations arise from time fluctuations in the flow, since the measurements were performed in the central region of the setup (of dimensions much larger than the Kolmogorov scale) where turbulence is expected to be more isotropic but large-scale fluctuations are likely to be strong as one impeller may temporarily dominate over others. Nonetheless, the value of $\mathcal{K}$ is compatible with zero, consistent with null mean helicity.

Helicity and the linking number of fluid trajectories in swirling flows. - The relation given by Eq. (2) holds for other turbulent flows besides HIT, and even locally in space, provided the region is large compared to the Kolmogorov scale, as $\mathcal{H}$ and $H$ are global, averaged quantities. We now consider the DNSs of TG turbulence and the VK laboratory experiments. Because of symmetries in the TG forcing $[38,44]$ the flow in these DNSs can be divided into eight cells each of volume $(\pi)^{3}$. The eight cells are labeled as $\left[C_{x}, C_{y}, C_{z}\right]$, where $C_{i}=1$ or 2 , with 1 labeling the region from 0 to $\pi$ in the $i$ th direction, and 2 the region between $\pi$ and $2 \pi$ (i.e., the cell labeled $[1,2,1]$ refers 
to the subregion $[0, \pi) \times[\pi, 2 \pi) \times[0, \pi)$ of the whole computational domain). Each cell has a flow that in many previous studies was shown to have Eulerian and Lagrangian similarities with that observed in VK experiments $[39,40,45]$, despite differences in boundary conditions and forcing mechanisms (volumetric forcing in the former, and two counterrotating propellers in the latter): two counterrotating vortices separated by a shear layer. The VK flow has nonzero helicity, while given the TG symmetries, four of the DNS cells have mean helicity with a preferential sign, and the other four cells have the opposite sign.

Figure 2(b) shows $\mathcal{K}$ for the VK experiment (with $\mathcal{H}$ estimated from DNSs and large-scale flow geometry), and for each cell in the TG simulations, as a function of each $\mathcal{H}$ value, for two different Reynolds numbers in $512^{3}$ or $1024^{3}$ TG DNSs. As before, in simulations $N=250$ and $\Delta T=\tau_{L}$. Fluctuations of $\mathcal{K}$ and $\mathcal{H}$ are larger than in HIT as this flow can have wild variations of helicity with time. However, a linear relation between both quantities is again recovered. A weighted least-squares fit using both resolutions yields $\alpha_{\mathrm{TG}}=0.23 \pm 0.01 \quad(95 \%$ confidence interval). A straight line with this slope is indicated as a reference in Fig. 2(b), as well as another with slope $\alpha_{\mathrm{HIT}}$ for comparison; $\alpha_{\mathrm{HIT}}$ and $\alpha_{\mathrm{TG}}$ are compatible within error bars (thus, in the following $\alpha=\alpha_{\mathrm{HIT}}$ ). This also shows that the linking number of fluid trajectories in subregions of the flow (as the individual cells in the DNSs of TG) is proportional to the local flow helicity. Figure 3(a) depicts the value of $\mathcal{K}(t)$ for each TG cell in simulations with $1024^{3}$ grid points. For each cell, $\mathcal{K}$ was computed with $\Delta T=\tau_{L}$ over ten random subsets of trajectories (each with 250 trajectories). The different points in each vertical stripe correspond to $\mathcal{K}$ estimated for these ten subsets (in groups of connected points for subsets in the same cells), at different consecutive time intervals up to time $6 \tau_{L} . \mathcal{K}$ (and $H$ ) fluctuates strongly in time in each cell, but half the cells preferentially have $\mathcal{K}>0$, and the other half $\mathcal{K}<0$. Given a TG cell and a time interval $\Delta T$, by averaging over the ten subsets a mean value of $\mathcal{K}$ at said time interval is computed, and a time series $\mathcal{K}(t)$ for each cell is thus reconstructed. Figure 3(b) shows the resulting time series of the linking number (or, except for the factor $1 / \alpha$, the flow's normalized helicity) for the cells with negative helicity.

The mean value of $\mathcal{K}$ in the $\mathrm{VK}$ experiment at a similar Reynolds number as the DNSs, obtained from PTV, is also shown in Fig. 3(a) by the dashed line, with a shaded horizontal stripe indicating $95 \%$ of the fluctuations. Figure 3(c) shows the PDF of $\mathcal{K}$ in the VK experiment for 500 measurements (the vertical axis is shared by both panels), and a normal distribution with the same mean and standard deviation. The value of $\mathcal{K}$ in the VK experiment and the TG cells with positive helicity are compatible within uncertainties, as expected from the similarities both flows share in their large-scale geometry, and despite the

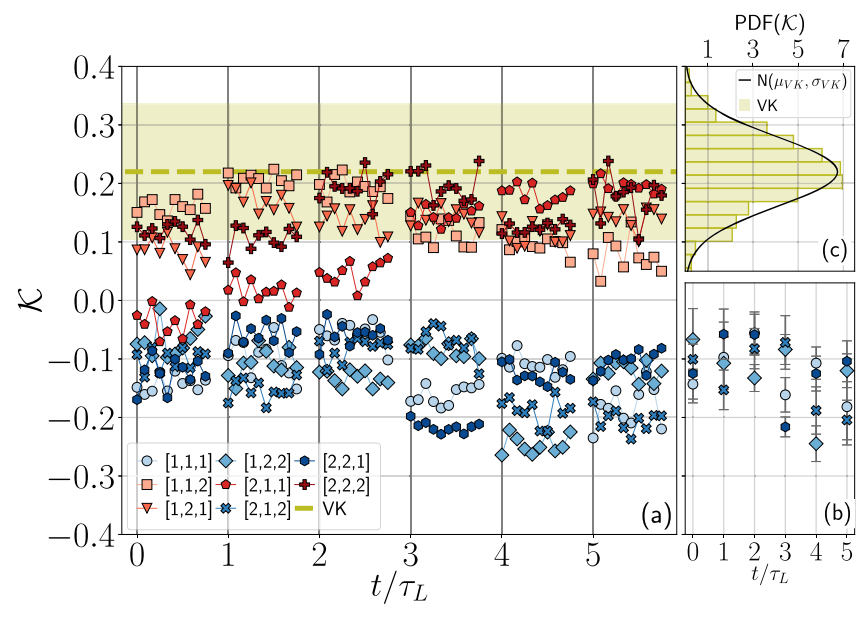

FIG. 3. (a) Linking number $\mathcal{K}$ as a function of time in the eight $\mathrm{TG}$ cells, and in the VK experiment. The different points correspond to $\mathcal{K}$ estimated for ten subsets of 250 particles (in groups of connected points), at different consecutive time intervals $\Delta T=\tau_{L}$ up to time $6 \tau_{L}$. Half the cells have $\mathcal{K}>0$ (with fluctuations in time), and the other half $\mathcal{K}<0$. The dashed line is the mean value of $\mathcal{K}$ in the $\mathrm{VK}$ experiment, with the colored band representing a $95 \%$ confidence interval considering time fluctuations. (b) Time evolution of $\mathcal{K}$ for all the cells with negative helicity, reconstructed by averaging the ten subsets from the data in (a). (c) PDF of $\mathcal{K}$ fluctuations in the VK experiment, with a normal distribution $N\left(\mu_{\mathrm{VK}}, \sigma_{\mathrm{VK}}\right)$ with the same mean $\mu_{\mathrm{VK}}$ and standard deviation $\sigma_{\mathrm{VK}}$ for comparison.

differences in boundary conditions and forcing mechanisms. Thus, the flow helicity has an imprint in the number of crossings of particles, and for very different turbulent flows, with or without mean flows, and with different boundaries.

We showed that fluid elements tell a story of the topology of the underlying turbulent flow. It is known that the irreversibility of turbulence has an effect on trajectories [46]. Here, the flow topology affects particles by linking the trajectories. In a mirror-symmetric flow, signed crossings average to zero. In a chiral flow that is not the case: flows with positive helicity result in a positive average of signed trajectory crossings, while flows with negative helicity result in negative signed crossings. Moreover, when properly normalized these two quantities are linearly related, with a proportionality constant that appears independent of the Reynolds number, the boundary conditions, and the mechanism that generates the turbulence. The linking of the particle paths involves macroscopic length scales and timescales. This is a remarkable feature, as a particle might go through flow regions in which strong fluctuations could potentially erase the broken mirror symmetry of the flow, especially in the TG flow. Statistical alignment between $\mathbf{u}$ and $\boldsymbol{\nabla} \times \mathbf{u}$, which may differ depending on flow helicity and was reported to take place preferentially in regions of low dissipation [47-51], can only partially explain these observations. As the particles move following $\mathbf{u}$, they may 
also partially follow vorticity field lines in helical regions, but this can only happen in such specific regions. The relation between $\mathcal{H}$ and $\mathcal{K}$ for long times and large scales thus indicates a stronger, and nontrivial, impact of flow chirality in physical observables and in the memory of fluid particles. The particle linking number thus defined connects a global topological invariant of the flow with a topological number of fluid trajectories. Moreover, the ratio of proportionality $\alpha$ is the same for very different flows, and in particular, for cases with helicity in the large-scale mean flow (i.e., TG and VK flows) as for HIT without a mean flow, both numerically and experimentally. This suggests that $\alpha$ may characterize a universal property of turbulence. Such connection has implications, e.g., for studies of mixing. If a particle's trajectories get more linked in a helical flow, then helicity can have an effect in mixing, something already noted in studies of helical flows in biological systems [52,53]. Finally, the relation between $\mathcal{H}$ and $\mathcal{K}$ provides a way to estimate helicity in laboratory experiments, a quantity which so far has eluded detailed laboratory characterization in turbulent flows. Indeed, one motivation to use small sets of particles or short trajectories, instead of the millions of long trajectories usually accessible in DNSs, was to probe the robustness of the particle linking number when used in conditions as those found in the laboratory.

This work was partially supported by the ECOS Project No. A18ST04. S. A., P. J. C., and P. D. M. acknowledge support from Grants Proyectos de Investigación Científica y Tecnológica No. 2015-3530 and No. 2018-4298, and UBACyT No. 20020170100508. M. B., S. G. H., and R. V. acknowledge support from European Project EuHIT (European High-Performance Infrastructures in Turbulence, Grant No. 312778), and ANR-13-BS09-0009. Computational resources were provided by the HPC center DIRAC, funded by Instituto de Fisica de Buenos Aires (UBACONICET) and the Sistema Nacional de Computación de Alto Desempeño - Ministerio de Ciencia, Tecnología e Innovación (Argentina) initiative.

* Corresponding author. sangriman@df.uba.ar

[1] J. M. Kosterlitz, Kosterlitz-Thouless physics: a review of key issues, Rep. Prog. Phys. 79, 026001 (2016).

[2] E. Fradkin, Field Theories of Condensed Matter Physics (Cambridge University Press, Cambridge, England, 2013), https://www.cambridge.org/academic/subjects/physics/ condensed-matter-physics-nanoscience-and-mesoscopicphysics/field-theories-condensed-matter-physics-2ndedition.

[3] L. H. Kauffman, Knots and Physics (World Scientific, Singapore, 2001), 10.1142/8338.

[4] H. Kedia, I. Bialynicki-Birula, D. Peralta-Salas, and W. T. M. Irvine, Tying Knots in Light Fields, Phys. Rev. Lett. 111, 150404 (2013).
[5] A. V. Vologodskii, N. J. Crisona, B. Laurie, P. Pieranski, V. Katritch, J. Dubochet, and A. Stasiak, Sedimentation and electrophoretic migration of DNA knots and catenanes, J. Mol. Biol. 278, 1 (1998).

[6] D. Krepel, A. Davtyan, N. P. Schafer, P. G. Wolynes, and J. N. Onuchic, Braiding topology and the energy landscape of chromosome organization proteins, Proc. Natl. Acad. Sci. U.S.A. 117, 1468 (2020).

[7] H. Moffatt and A. Tsinober, Helicity in laminar and turbulent flow, Annu. Rev. Fluid Mech. 24, 281 (1992).

[8] H. K. Moffatt, Helicity and singular structures in fluid dynamics, Proc. Natl. Acad. Sci. U.S.A. 111, 3663 (2014).

[9] A. Enciso, D. Peralta-Salas, and F. T. de Lizaur, Helicity is the only integral invariant of volume-preserving transformations, Proc. Natl. Acad. Sci. U.S.A. 113, 2035 (2016).

[10] A. Pouquet, U. Frisch, and J. Lorat, Strong MHD helical turbulence and the nonlinear dynamo effect, J. Fluid Mech. 77, 321 (1976).

[11] N. Yokoi and A. Yoshizawa, Statistical analysis of the effects of helicity in inhomogeneous turbulence, Phys. Fluids A 5, 464 (1993).

[12] A. Brandenburg and K. Subramanian, Astrophysical magnetic fields and nonlinear dynamo theory, Phys. Rep. 417, 1 (2005).

[13] K. Inagaki, N. Yokoi, and F. Hamba, Mechanism of mean flow generation in rotating turbulence through inhomogeneous helicity, Phys. Rev. Fluids 2, 114605 (2017).

[14] E. N. Rasmussen and D. O. Blanchard, A baseline climatology of sounding-derived supercell and tornado forecast parameters, Weather Forecast. 13, 1148 (1998).

[15] C. Rorai, D. Rosenberg, A. Pouquet, and P. D. Mininni, Helicity dynamics in stratified turbulence in the absence of forcing, Phys. Rev. E 87, 063007 (2013).

[16] R. Marino, P. D. Mininni, D. Rosenberg, and A. Pouquet, Emergence of helicity in rotating stratified turbulence, Phys. Rev. E 87, 033016 (2013).

[17] G. P. Bewley, M. S. Paoletti, K. R. Sreenivasan, and D. P. Lathrop, Characterization of reconnecting vortices in superfluid helium, Proc. Natl. Acad. Sci. U.S.A. 105, 13707 (2008)

[18] C. Rorai, K. R. Sreenivasan, and M. E. Fisher, Propagating and annihilating vortex dipoles in the Gross-Pitaevskii equation, Phys. Rev. B 88, 134522 (2013).

[19] D. S. Hall, M. W. Ray, K. Tiurev, E. Ruokokoski, A. H. Gheorghe, and M. Möttönen, Tying quantum knots, Nat. Phys. 12, 478 (2016).

[20] M. C. Tsatsos, P. E. Tavares, A. Cidrim, A. R. Fritsch, M. A. Caracanhas, F. E. A. dos Santos, C. F. Barenghi, and V. S. Bagnato, Quantum turbulence in trapped atomic BoseEinstein condensates, Phys. Rep. 622, 1 (2016).

[21] P. Clark di Leoni, P. D. Mininni, and M. E. Brachet, Helicity, topology, and Kelvin waves in reconnecting quantum knots, Phys. Rev. A 94, 043605 (2016).

[22] H. Kedia, D. Kleckner, M. W. Scheeler, and W. T. M. Irvine, Helicity in superfluids: Existence and the classical limit, Phys. Rev. Fluids 3, 104702 (2018).

[23] E. Herbert, F. Daviaud, B. Dubrulle, S. Nazarenko, and A. Naso, Dual non-Kolmogorov cascades in a von Kármán flow, Europhys. Lett. 100, 44003 (2012). 
[24] P. D. Mininni, A. Alexakis, and A. Pouquet, Scale interactions and scaling laws in rotating flows at moderate Rossby numbers and large Reynolds numbers, Phys. Fluids 21, 015108 (2009).

[25] G. Sahoo, A. Alexakis, and L. Biferale, Discontinuous Transition from Direct to Inverse Cascade in Three-Dimensional Turbulence, Phys. Rev. Lett. 118, 164501 (2017).

[26] R. H. Kraichnan and R. Panda, Depression of nonlinearity in decaying isotropic turbulence, Phys. Fluids 31, 2395 (1988).

[27] A. Cameron, A. Alexakis, and M.-E. Brachet, Effect of helicity on the correlation time of large scales in turbulent flows, Phys. Rev. Fluids 2, 114602 (2017).

[28] L. Wasserman, Topological data analysis, Annu. Rev. Stat. Appl. 5, 501 (2018).

[29] M. W. Scheeler, D. Kleckner, D. Proment, G. L. Kindlmann, and W. T. M. Irvine, Helicity conservation by flow across scales in reconnecting vortex links and knots, Proc. Natl. Acad. Sci. U.S.A. 111, 15350 (2014).

[30] M. W. Scheeler, W. M. van Rees, H. Kedia, D. Kleckner, and W. T. M. Irvine, Complete measurement of helicity and its dynamics in vortex tubes, Science 357, 487 (2017).

[31] K. Gustavsson and L. Biferale, Preferential sampling of helicity by isotropic helicoids, Phys. Rev. Fluids 1, 054201 (2016).

[32] Y. Kimura and H. K. Moffatt, Reconnection of skewed vortices, J. Fluid Mech. 751, 329 (2014).

[33] C. E. Laing, R. L. Ricca, and D. W. L. Sumners, Conservation of writhe helicity under anti-parallel reconnection, Sci. Rep. 5, 9224 (2015).

[34] See Supplemental Material at http://link.aps.org/ supplemental/10.1103/PhysRevLett.127.254502 for details on the simulations and experiments, and on analysis on the robustness of the results.

[35] P. D. Mininni, D. Rosenberg, R. Reddy, and A. Pouquet, A hybrid MPI-OpenMP scheme for scalable parallel pseudospectral computations for fluid turbulence, Parallel Comput. 37, 316 (2011).

[36] D. Rosenberg, P. D. Mininni, R. Reddy, and A. Pouquet, GPU parallelization of a hybrid pseudospectral geophysical turbulence framework using CUDA, Atmosphere 11, 178 (2020).

[37] A. Pouquet and G. S. Patterson, Numerical simulation of helical magnetohydrodynamic turbulence, J. Fluid Mech. 85, 305 (1978).

[38] M. E. Brachet, D. I. Meiron, S. A. Orszag, B. G. Nickel, R. H. Morf, and U. Frisch, Small-scale structure of the Taylor-Green vortex, J. Fluid Mech. 130, 411 (1983).

[39] Y. Ponty, P. D. Mininni, D. C. Montgomery, J.-F. Pinton, H. Politano, and A. Pouquet, Numerical Study of Dynamo Action at Low Magnetic Prandtl Numbers, Phys. Rev. Lett. 94, 164502 (2005).
[40] S. Angriman, P. D. Mininni, and P. J. Cobelli, Velocity and acceleration statistics in particle-laden turbulent swirling flows, Phys. Rev. Fluids 5, 064605 (2020).

[41] R. Zimmermann, H. Xu, Y. Gasteuil, M. Bourgoin, R. Volk, J.-F. Pinton, and E. Bodenschatz, The Lagrangian exploration module: An apparatus for the study of statistically homogeneous and isotropic turbulence, Rev. Sci. Instrum. 81, 055112 (2010).

[42] M. Bourgoin and S. G. Huisman, Using ray-traversal for 3D particle matching in the context of particle tracking velocimetry in fluid mechanics, Rev. Sci. Instrum. 91, 085105 (2020).

[43] B. L. Sawford and P. K. Yeung, Kolmogorov similarity scaling for one-particle Lagrangian statistics, Phys. Fluids 23, 091704 (2011).

[44] C. Nore, M. Brachet, H. Politano, and A. Pouquet, Dynamo action in the Taylor-Green vortex near threshold, Phys. Plasmas 4, 1 (1997).

[45] P. Mininni, P. Dmitruk, P. Odier, J.-F. Pinton, N. Plihon, G. Verhille, R. Volk, and M. Bourgoin, Long-term memory in experiments and numerical simulations of hydrodynamic and magnetohydrodynamic turbulence, Phys. Rev. E 89, 053005 (2014).

[46] H. Xu, A. Pumir, G. Falkovich, E. Bodenschatz, M. Shats, H. Xia, N. Francois, and G. Boffetta, Flight-crash events in turbulence, Flight-crash events in turbulence, Proc. Natl. Acad. Sci. U.S.A. 111, 7558 (2014).

[47] R. B. Pelz, V. Yakhot, S. A. Orszag, L. Shtilman, and E. Levich, Velocity-Vorticity Patterns in Turbulent Flow, Phys. Rev. Lett. 54, 2505 (1985).

[48] H. K. Moffatt, Magnetostatic equilibria and analogous Euler flows of arbitrarily complex topology. Part 1. Fundamentals, J. Fluid Mech. 159, 359 (1985).

[49] L. Shtilman, E. Levich, S. A. Orszag, R. B. Pelz, and A. Tsinober, On the role of helicity in complex fluid flows, Phys. Lett. 113A, 32 (1985).

[50] E. Levich, Certain problems in the theory of developed hydrodynamical turbulence, Phys. Rep. 151, 129 (1987).

[51] M. Farge, G. Pellegrino, and K. Schneider, Coherent Vortex Extraction in 3D Turbulent Flows Using Orthogonal Wavelets, Phys. Rev. Lett. 87, 054501 (2001).

[52] R. E. Goldstein, I. Tuval, and J.-W. van de Meent, Microfluidics of cytoplasmic streaming and its implications for intracellular transport, Proc. Natl. Acad. Sci. U.S.A. 105, 3663 (2008).

[53] J.-W. Van de Meent, A. J. Sederman, L. F. Gladden, and R. E. Goldstein, Measurement of cytoplasmic streaming in single plant cells by magnetic resonance velocimetry, J. Fluid Mech. 642, 5 (2010). 